\title{
ON APPROXIMATION CONDITIONS OF THE DISTRIBUTION OF THE MAXIMUM SUMS OF INDEPENDENT RANDOM VARIABLES
}

\section{A.N. Startsev}

Let $\xi_{n i}, i=1, \ldots, n, n \geq 1$ be an array of row-independent random variables with $\mathbf{E} \xi_{n i}=a_{n i}$ and $\mathbf{D} \xi_{n i}=\sigma_{n i}^{2}$. We can assume, without any loss of generality, that $\sigma_{n 1}^{2}+\cdots+\sigma_{n n}^{2}=1$ for every $n \geq 1$.

Define, with an abuse of notation,

$$
t_{0}=S_{0}=0, \quad S_{k}=\sum_{i=1}^{k} \xi_{n i}, \quad t_{k}=\sum_{i=1}^{k} \sigma_{n i}^{2}, \quad \bar{S}_{n}=\max \left(S_{n 1}, \ldots, S_{n n}\right) .
$$

We are interested in conditions for $\bar{S}_{n}-A_{n}$ to converge weakly to some limit law; here $A_{n}$ is some centering sequence.

For identically distributed random variables $\xi_{n i}$ with $\sigma_{n i}=n^{-1}, i=1, \ldots, n$, such conditions were found by A. Wald [14], who established the following results:

(i) If $\lim _{n \rightarrow \infty} n a_{n 1}=a, 0 \leq a<\infty$, then for any $x>0$

$$
\lim _{n \rightarrow \infty} P\left(\bar{S}_{n}<x\right)=W_{a}(x) \equiv \frac{a x}{2 \sqrt{\pi}} e^{a x} \int_{a^{2} / 2}^{\infty} u^{-3 / 2} \exp \left(-\frac{a^{2} x^{2}}{4 u}-u\right) d u
$$

where, in particular $W_{0}(x)=\max (0,2 \Phi(x)-1)$;

(ii) If $\lim _{n \rightarrow \infty} n a_{n 1}=+\infty$, then for any $x \in \mathbf{R}$

$$
\lim _{n \rightarrow \infty} P\left(\bar{S}_{n}-n a_{n 1}<x\right)=\Phi(x) \equiv(2 \pi)^{-1 / 2} \int_{-\infty}^{x} \exp \left(-u^{2} / 2\right) d u .
$$

Analogous results in the case of identically distributed random variables, but in a more general situation, were obtained by A.A. Borovkov [2].

K.L. Chung [3] extended the results of A. Wald to the case of nonidentically distributed random variables having common means and variances. He obtained in addition the rate of convergence of the order $n^{-1 / 26} \ln n$ when $\mathbf{E}\left|\xi_{n i}-a_{n i}\right|^{3} \leq$ $C<\infty$ for all $i=1, \ldots, n$ and $n \geq 1$. 
V.B. Nevzorov [6] obtained more general conditions for the convergence to the normal law in the scheme $\xi_{n i}=\xi_{i} / B_{n}$, where $B_{n}^{2}=\sum_{i=1}^{n} \mathbf{D} \xi_{i}$. He proved that the conditions

(a) $\left\{\xi_{i}: i \geq 1\right\}$ satisfy the central limit theorem;

(b) $\lim _{n \rightarrow \infty} \sqrt{n} \min \left(\mathbf{E} \xi_{1}, \ldots, \mathbf{E} \xi_{n}\right)=+\infty$;

(c) $\mathbf{D} \xi_{i} \leq D<\infty, \quad B_{k}^{2}>d k, \quad d>0, \quad k=1,2, \ldots$,

are sufficient for the weak convergence of the random variables $\zeta_{n}^{*}=\left(\max _{1 \leq k \leq n} \zeta_{k}-\right.$ $\left.\mathbf{E} \zeta_{n}\right) B_{n}^{-1}$ to the normal law; here $\zeta_{k}=\xi_{1}+\xi_{2}+\cdots+\xi_{k}, k=1,2, \ldots$.

In our notation the conditions (b) and (c) take the following form

$$
\begin{aligned}
& \lim _{n \rightarrow \infty} n \min _{1 \leq i \leq n} a_{n i}=+\infty, \quad \sigma_{n k}^{2} \leq C_{1} n^{-1}, \\
& t_{k} \geq C_{2} k n^{-1}, \quad 0<C_{i}<\infty, \quad i=1,2, \quad k=1, \ldots, n, \quad n \geq 1 .
\end{aligned}
$$

Nevzorov constructed examples which show that it is not possible to take conditions ( $\left.\mathrm{b}^{\prime}\right) \mathbf{E} \zeta_{k} \geq C k, C>0, k \geq 1$ and $\left(\mathrm{c}^{\prime}\right) C_{1} k \leq B_{k}^{2} \leq C_{2} k, k \geq 1,0<$ $C_{1}<C_{2}<\infty$ instead of (b) and (c), respectively.

We shall give only one of these examples since the other one is rather exotic: random variables satisfy the central limit theorem but not the Lindeberg condition.

Example. Let $\left\{\eta_{i}, i \geq 1\right\}$ be independent standard normally distributed random variables. Define

$$
\xi_{k}=\left\{\begin{array}{ll}
\eta_{k}-k / 2, & k=2^{m} \\
\eta_{k}+6, & k \neq 2^{m}
\end{array} \quad, \text { when } m=1,2, \ldots\right.
$$

It is easy to check that the conditions (a) and (b) are satisfied and $\mathbf{E} \zeta_{n}=5 n-$ $6 \log _{2} n$ when $n=2^{m}$, but

$$
P\left(\zeta_{n}^{*}<0\right) \longrightarrow 0 \neq \Phi(0), \quad \text { as } n \rightarrow \infty .
$$

In spite of this, the convergence to the normal law takes place for $n=2^{m}-1$. The reason for this phenomenon will be evident later.

In a recent paper V.M. Kruglov [4] obtained necessary and sufficient conditions for the convergence of the distribution of $\bar{S}_{n}$ to the limit laws $W_{a}$ and $\Phi$ in the general scheme of partial sums, both under moment restrictions and without them, but the convergence to the normal law under moment restrictions is considered only for non-negative means.

In the present paper sufficient conditions for the convergence of the distribution of $\bar{S}_{n}$ to the normal law and to other limit laws are given in a more general case by the method of approximation of the Wiener process (the invariance principle). A similar approach was used by A.V. Nagaev and the author [5], [12] in 
an asymptotical analysis of mathematical models of epidemics, exactly as by A.A. Borovkov [2] in an analysis of this problem in the case of identically distributed random variables and by Yu. V. Prohorov [9] in an investigation of transition phenomena in the queueing process and then by V.B. Nevzorov [7] in a research of limit distributions of various order statistics of successive sums of random variables, but with zero means.

Let us pass to the formulation of the results obtained. It is assumed that $\left\{\xi_{n i}: 1 \leq i \leq n\right\}$ satisfy the Lindeberg condition.

Define $A_{k} \equiv \mathrm{E} S_{k}=a_{n 1}+a_{n 2}+\cdots+a_{n k}, k=1, \ldots, n, n \geq 1$.

Theorem 1. Let $a_{n k} \geq 0, n_{1}<k \leq n$. If $\lim _{n \rightarrow \infty} t_{n_{1}}=1$ and if $\lim _{n \rightarrow \infty} \min _{1 \leq k \leq n_{1}}\left(A_{n}-A_{k}\right)=+\infty$, then

$$
\lim _{n \rightarrow \infty} P\left(\bar{S}_{n}-A_{n}<x\right)=\Phi(x) \text { for all } x \in \mathbf{R} .
$$

Remark. We want to point out that, if $k=[n \alpha], 1 / 2<\alpha<1$, then under the conditions of the previous example one has

$$
A_{n}-A_{k} \leq-\sqrt{n}(1 / 2-6(1-\alpha)) \longrightarrow-\infty, \quad \text { if } \alpha>11 / 12
$$

We claim that $P\left(\bar{S}_{n}-A_{n}<x\right) \rightarrow 0$, but this follows at once from the fact that $a_{n n}=-\sqrt{n} / 2 \rightarrow-\infty$. In fact,

$$
P\left(\bar{S}_{n}-A_{n}<x\right) \leq P\left(S_{n-1}-A_{n-1}<x+a_{n n}\right)=\Phi(x-\sqrt{n} / 2)+o(1)=o(1) .
$$

It is not difficult to show that the conditions of Theorem 1 will hold for $n=2^{m}-1$.

Corollary 1. Let $a_{n k} \geq 0, k=1, \ldots, n$. If there exists $n_{1}<n$ such that $\lim _{n \rightarrow \infty} t_{n_{1}}=1, \lim _{n \rightarrow \infty}\left(A_{n}-A_{n_{1}}\right)=+\infty$, then $\lim _{n \rightarrow \infty} P\left(\bar{S}_{n}-A_{n}<x\right)=$ $\Phi(x)$ for all $x \in \mathbf{R}$.

This statement is the same as the sufficiency part of Theorem 7 by Kruglov [4].

Now we are going to give results where the limit law is not a normal one.

Theorem 2. If $\lim _{n \rightarrow \infty} \max _{1 \leq k \leq n}\left|A_{k}-g\left(t_{k}\right)\right|=0$, where $g(t)$ satisfies a Lipschitz condition and $g(0)=0$, then for any $x \in \mathbf{R}^{+}$

$$
\lim _{n \rightarrow \infty} P\left(\bar{S}_{n}<x\right)=P\left(\max _{0 \leq t \leq 1}(w(t)+g(t))<x\right),
$$

where $w(t)$ stands for the standard Wiener process on $[0,1]$.

Corollary 2. If $\lim _{n \rightarrow \infty} \max _{1 \leq k \leq n}\left|A_{k}\right|=0$ (i.e., $g(t) \equiv 0$ ), then for any $x \in \mathbf{R}^{+}$

$$
\lim _{n \rightarrow \infty} P\left(\bar{S}_{n}<x\right)=W_{0}(x) \equiv P\left(\max _{0 \leq t \leq 1} w(t)<x\right) .
$$


This result follows also from Theorem 4 by Kruglov [4] and Theorem 10 by Rényi [10], where it is supposed that $a_{n i}=0, i=1, \ldots, n$.

Corollary 3. Let $a_{n k}=a_{n}, \sigma_{n k}^{2}=n^{-1}, k=1, \ldots, n, n \geq 1$. If $\lim _{n \rightarrow \infty} n a_{n}=a,|a|<\infty$, then for any $x>0$

$$
\begin{aligned}
\lim _{n \rightarrow \infty} P\left(\bar{S}_{n}<x\right) & =P(w(t)<x-a t, 0 \leq t \leq 1) \\
& \equiv 1-\exp \{-x(|a|-a)\} \int_{0}^{a^{2} / 2} p_{(x a / 2)^{2}}(u) d u
\end{aligned}
$$

where $p_{q}(u)=\sqrt{q /\left(\pi u^{3}\right)} \exp (2 \sqrt{q}-u-q / u), u>0, q>0$, is the density function of the Wald distribution.

This statement follows also from Theorem 6 by Kruglov [4], where the existence of the limits

$$
\lim _{n \rightarrow \infty} \min _{1 \leq i \leq n} \frac{a_{n i}}{\sigma_{n i}^{2}}=\lim _{n \rightarrow \infty} \max _{1 \leq i \leq n} \frac{a_{n i}}{\sigma_{n i}^{2}}=a
$$

is required but the explicit form of the limit law is given in the case $a>0$ only. The explicit form of this limit law is given by Borovkov [2], but his result was obtained in the case of identically distributed random variables under the condition that the sequence $a_{n}$ tends to zero without change of the sign.

If $a>0$, then we obtain the result of Wald [14]:

$$
\lim _{n \rightarrow \infty} P\left(\bar{S}_{n}<x\right)=W_{a}(x) \equiv \int_{a^{2} / 2}^{\infty} p_{(x a / 2)^{2}}(u) d u
$$

Proof of Theorem 1. Define

$$
P_{n}(x)=P\left(\bar{S}_{n}-A_{n}<x\right)=P\left(S_{k}^{0}<A_{n}-A_{k}+x, k=1, \ldots, n\right),
$$

where $S_{k}^{0}=S_{k}-A_{k}, k=1, \ldots, n$.

Let $s_{n}(t), 0 \leq t \leq 1$, be the random polygonal line constructed by using the points $\left(t_{k}, S_{k}^{0}\right)$ and $g_{n}(t)$ the polygon with vertices in the points $\left(t_{k}, A_{n}-A_{k}\right), k=$ $0,1, \ldots, n$.

Then we can rewrite (1) into the form

$$
P_{n}(x)=P\left(s_{n}(t)<g_{n}(t)+x, 0 \leq t \leq 1\right) .
$$

According to the invariance principle it is possible to construct $s_{n}(t)$ and $w(t)$ on the same probability space such that (cf. [1])

$$
s_{n}(t)=w(t)+\varepsilon_{n}(t)
$$


where the random process $\varepsilon_{n}(t)$ satisfies the following condition

$$
\varepsilon_{n} \equiv \sup _{0 \leq t \leq 1}\left|\varepsilon_{n}(t)\right| \stackrel{\mathrm{P}}{\longrightarrow} 0 \text { as } n \rightarrow \infty .
$$

Now it is not difficult to show that (2)-(4) imply the inequality

$$
P_{n}(x) \geq P\left(w(t)<g_{n}(t)+x-\varepsilon, 0 \leq t \leq 1\right)+\delta_{n},
$$

for any $\varepsilon>0$, where $\delta_{n}=O\left(P\left(\varepsilon_{n}>\varepsilon\right)\right)$.

On the other hand by virtue of the central limit theorem

$$
P_{n}(x) \leq P\left(S_{n}^{0}<x\right)=\Phi(x)+o(1) .
$$

After defining the following events

$A=\left\{w(t)<g_{n}(t)+x-\varepsilon, 0 \leq t \leq t_{n_{1}}\right\}, \quad B=\left\{w(t)<g_{n}(t)+x-\varepsilon, t_{n_{1}}<t \leq 1\right\}$

we can rewrite (5) into the form

$$
P_{n}(x) \geq P(B)-P(B \bar{A})+\delta_{n}
$$

Lemma. If $h_{n}(t)$ is a non-increasing function for $t \in\left(\tau_{n}, 1\right], \lim _{n \rightarrow \infty} \tau_{n}=1$ and $\lim _{n \rightarrow \infty} h_{n}(1)=h_{0}$, then

$$
Q_{n} \equiv P\left(w(t)<h_{n}(t), \tau_{n}<t \leq 1\right) \longrightarrow \Phi\left(h_{0}\right), \quad \text { as } n \rightarrow \infty .
$$

Proof of Lemma. It is easy to see that

$$
Q_{n} \leq P\left(w(1)<h_{n}(1)\right) \longrightarrow \Phi\left(h_{0}\right) .
$$

Since $h_{n}(t)$ is a non-increasing function, we have

$$
\begin{aligned}
Q_{n} & \geq P\left(w(t)<h_{n}(1), \tau_{n}<t \leq 1\right) \\
& =P\left(\max _{0 \leq t \leq 1-\tau_{n}} w(t)+w\left(\tau_{n}\right)<h_{n}(1)\right) \longrightarrow \Phi\left(h_{0}\right) .
\end{aligned}
$$

Here we have used the fact that

$$
\max _{0 \leq t \leq 1-\tau_{n}} w(t) \stackrel{\mathrm{P}}{\longrightarrow} 0, \quad w\left(\tau_{n}\right) \stackrel{\mathrm{P}}{\longrightarrow} w(1), \text { as } \mathrm{n} \rightarrow \infty .
$$

Clearly, (8) and (9) together give then the statement of the lemma. 
We are now returning to the proof of Theorem 1. By the lemma and the conditions on $g_{n}(t)$ we can obtain

$$
P(B)=\Phi(x-\varepsilon)+o(1) .
$$

Now we will show that $P(\bar{A})=o(1)$. In fact

$$
\begin{aligned}
P(A) & \geq P\left(w(t)<\min _{0 \leq t \leq t_{n_{1}}} g_{n}(t)+x-\varepsilon, 0 \leq t \leq t_{n_{1}}\right) \\
& \geq P\left(\max _{0 \leq t \leq 1} w(t)<\min _{0 \leq t \leq t_{n_{1}}} g_{n}(t)+x-\varepsilon\right) \\
& =1-2 P\left(w(1) \geq \min _{0 \leq t \leq t_{n_{1}}} g_{n}(t)+x-\varepsilon\right)=1+o(1),
\end{aligned}
$$

since $\min _{0 \leq t \leq t_{n_{1}}} g_{n}(t)=\min _{0 \leq k \leq n_{1}}\left(A_{n}-A_{k}\right) \rightarrow \infty$ as $n \rightarrow \infty$.

The statement of Theorem 1 follows then from (1), (6), (7), (10) and (11).

Proof of Theorem 2. It is easy to see, by using the preceding arguments, that for any $\varepsilon>0$

$$
\begin{aligned}
& P\left(w(t)<g(t)+x-\varepsilon-\Delta_{n}, \quad 0 \leq t \leq 1\right)-\delta_{n} \leq P\left(\bar{S}_{n}<x\right) \\
& \leq P\left(w(t)<g(t)+x+\varepsilon+\Delta_{n}, \quad 0 \leq t \leq 1\right)+\delta_{n},
\end{aligned}
$$

where $\Delta_{n}=\max _{1 \leq k \leq n}\left|A_{k}-g\left(t_{k}\right)\right|, \delta_{n}=O\left(P\left(\varepsilon_{n}>\varepsilon\right)\right)$.

The statement of Theorem 2 follows then from the inequality (see [8] or [9])

$$
|P(w(t)<g(t)+h, 0 \leq t \leq 1)-P(w(t)<g(t), 0 \leq t \leq 1)| \leq C h .
$$

In order to prove Corollary 3 we remark that L. Takács [13] has obtained the following result (see also Borovkov [2])

$$
P(w(t)<x-a t, 0 \leq t \leq T)=1-x \int_{0}^{T} \Phi^{\prime}\left(\frac{x-a t}{\sqrt{t}}\right) t^{-3 / 2} d t
$$

implying the claimed form of this probability.

Acknowledgement. The author thanks the referee whose remarks have helped to improve this paper.

\section{References}

[1] BorISOv, I.S., and A.A. Borovkov: Second-order approximation of random polygonal lines in the Donsker-Prokhorov invariance principle. - Theory Probab. Appl. 31, 1987, 179-202 (English translation of Teor. Veroyatnost. i Primenen. 31, 1986, 225-245).

[2] Borovkov, A.A.: Stochastic processes in queueing theory. - Springer-Verlag, New YorkHeidelberg-Berlin, 1976 (English translation). 
[3] Chung, K.L.: Asymptotic distribution of the maximum cumulative sums of independent random variables. - Bull. Amer. Math. Soc. 54, 1948, 1162-1170.

[4] KRUGLOV, V.M.: The central limit theorem and weak convergence of maxima of sums of independent random variables . - Stability Problems for Stochastic Models, Proceedings, Uzhgorod 1984, V.V. Kalashnikov and V.M. Zolotarev (Eds.), Lecture Notes in Mathematics 1155, - Springer-Verlag, Berlin-Heidelberg-New York-Tokyo, 144-179, 1985.

[5] Nagaev, A.V., and A.N. Startsev: The asymptotic analysis of a stochastic model of an epidemic. - Theory Probab. Appl. 15, 1970, 98-107 (English translation of Teor. Veroyatnost. i Primenen. 15, 1970, 97-105).

[6] Nevzorov, V.B.: The convergence of the distribution of the maximum of successive sums of independent random variables to a normal law. - Vestnik Leningrad. Univ. Mat. Mekh. Astronom. 1972, 1, 34-44 (Russian).

[7] Nevzorov, V.B.: Order statistics constructed from sums of random variables. - Theory Probab. Appl. 30, 1986, 211-212 (English translation of Teor. Veroyatnost. i Primenen. 30, 1985, 193-194).

[8] Prokhorov, YU. V.: Convergence of random processes and limit theorems in probability theory. - Theory Probab. Appl. 1, 1956, 157-214 (English translation of Teor. Veroyatnost. i Primenen. 1, 1956, 177-238).

[9] Proknorov, Yu. V.: Transition phenomena in the queueing process I. - Litovsk. Mat. Sb. 3, 1963, 1, 199-205 (Russian).

[10] RÉNYI, A.: On the theory of order statistics. - Acta Math. Acad. Sci. Hungar. 4, 1953, 191-231.

[11] Skorohod, A.V.: Studies in the theory of random processes. - Addison-Wesley Publ. Co., Inc., Reading, Mass., 1965 (English translation).

[12] Startsev, A.N.: In: Random processes and statistical decisions. - Fan, Tashkent, 1974 (Russian).

[13] TAKÁCS, L.: Combinatorial methods in the theory of stochastic processes. - John Wiley \& Sons, Inc., New York-London-Sydney, 1967.

[14] WALD, A.: Limit distribution of the maximum and minimum of successive cumulative sums of random variables. - Bull. Amer. Math. Soc. 53, 1947, 142-153.

Academy of Sciences Uzbek SSR

Institute of Mathematics

F. Hodjaeva 29

700143 Tashkent

USSR 